\title{
Soroprevalência de aglutininas anti-Leptospira spp. em ovinos nas Mesorregiões Sudeste e Sudoeste do Estado Rio Grande do Sul, Brasil
}

\author{
Seroprevalence of agglutinins anti-Leptospira spp. in sheep from the Southeast and Southwest \\ Mesoregions of the State of Rio Grande do Sul, Brazil
}

\author{
Geder Paulo Herrmann' ${ }^{1}$ Andrey Pereira Lage ${ }^{2}$ Elvio Carlos Moreira² João Paulo Amaral Haddad ${ }^{2}$ \\ José Renato de Resende ${ }^{3}$ Rogério Oliveira Rodrigues ${ }^{4}$ Rômulo Cerqueira Leite ${ }^{2}$
}

\section{RESUMO}

A presença de aglutininas anti-Leptospira spp. em 1360 amostras soros de ovinos, clinicamente sadios com mais de um ano de idade, criados extensivamente em 136 fazendas de 18 municípios; 10 na Mesorregião Sudeste e 8 na Mesorregião Sudoeste do Estado do Rio Grande do Sul, entre os meses de janeiro a março de 1999. Foi determinado pela Técnica de Aglutinação Microscópica (MAT), das 1360 amostras de soros testados, 466 (34,26\%) animais foram reagentes $e$ os títulos de aglutininas anti-Leptospira spp. variavam de 100 a 3200. As sorovariedades encontradas foram: hardjo (Norma), 210 (28,4\%), sentot, 152 (16,8\%); hardjoprajitno, 133 (14,5\%); fortbragg 73 (6,3\%), wolffi, 39 (4,7\%); pyrogenes, 25 (1,8\%); australis, 21 (1,6\%); pomona, 20 (1,6\%); sejroe, 19 (2,2\%); castellonis, 18(1,8\%); hebdomadis, 17 (1,3\%); icterohaemorrhagiae, $16(0,5 \%)$; grippotyphosa, $9(0,7 \%)$; canicola, $8(0,6 \%)$; tarassovi, 7 (0,6\%), bratislava, 4 (0,29\%), autumnalis, 3 (0,2\%). Os resultados mostraram que as Leptospira spp. estão disseminadas na maioria das fazendas que criam ovinos nas Mesorregiões Sudeste e Sudoeste do Rio Grande do Sul.

Palavras-chave: leptospirose, ovino, prevalência, Rio Grande do Sul.

\section{ABSTRACT}

The presence of anti-Leptospira agglutinins in 1.360 samples of ovine sera was determined. Clinically healthy sheep with more than one year of age, raised in pasture in 136 farms of 18 counties, 10 located in the southeast Mesorregions and 8 in the southwest Mesorregions of the state of Rio Grande do Sul, Brazil, between the months of January and March of 1999. Were used sera tested by the Microscopic Agglutination Technique (MAT), from the 1.360 samples of serum tested, $466(34.26 \%)$ were positive and the titers of anti-Leptospira spp. agglutinins varied from 100 to 3.200. The serovars founded were: hardjo (Norma), 210 (28.4\%), sentot, 152 (16.8\%); hardjoprajitno, 133 (14.5\%); fortbragg, 73 (6.3\%); wolffi, 39 (4.7\%); pyrogenes, 25 (1.8\%); australis, 21 (1.6\%); pomona, 20 (1.6\%); sejroe, 19 (2.2\%); castellonis, 18 (1.8\%); hebdomadis, 17 (1.3\%); icterohaemorrhagiae, 16 (0.5\%); grippotyphosa, $9(0.7 \%)$; canicola , 8 (0.6\%); tarassovi, 7 (0.6\%), bratislava, 4 (0.29\%), autumnalis, $3(0.2 \%)$. The results demonstrate that Leptospira spp are disseminated in the majority of the farms that raise sheep in the southeast and southwest Mesorregions of the State of Rio Grande do Sul, Brazil.

Key words: leptospirosis, sheep, epidemiology, Rio Grande do Sul.

\section{INTRODUÇÃO}

O gênero Leptospira spp, família Leptospiraceae, recentemente foi classificado por estudos de afinidades antigênicas e análises moleculares, e os agentes etiológicos das leptospiroses foram divididos em duas espécies patogênicas e não patogênicas. As leptospiras patogênicas estão divididas em oito espécies, distribuídas em mais 200 sorovariedades e arranjadas em 23 sorogrupos (FAINE, 1999).

As Leptospiras patogênicas são agentes etiológicos de importância zoonótica para o homem e os animais, e já foram descritas em uma grande variedade de animais domésticos e selvagens, que

\footnotetext{
${ }^{1}$ Médico Veterinário, Doutor, Professor, Departamento de Microbiologia e Parasitologia, Universidade Federal de Santa Maria, Av. Roraima s/n. Campus Universitário, 97105-900, Santa Maria RSE-mail: gederpaulo@bol.com.br Autor para correspondência. ${ }^{2}$ Médico Veterinário, Doutor, Professor, Departamento de Medicina Veterinária Preventiva, Universidade Federal de Minas Gerais (UFMG), Belo Horizonte , MG.

${ }^{3}$ Médico Veterinário, Mestre, Prefeitura Municipal de Belo Horizonte, MG.

${ }^{4}$ Médico Veterinário, aluno do Programa de Pós-graduação, Escola de Veterinária, UFMG.
} 
adquirem naturalmente a infecção, no entanto um pequeno número de animais manifestam a doença. Os ovinos são os animais domésticos considerados menos susceptíveis, porém sofrem a infecção das leptospiras patogênicas e, em muitos casos, a evolução é assintomática, podendo, às vezes, ocorrerem surtos da doença com abortamento e morte de cordeiros. A leptospirose pode manifestar-se de forma aguda, subaguda e crônica, caracterizando-se por quadros clínicos de septicemia, hemorragia, nefrite, seguido por icterícia, hemoglobinúria, mastite sanguinolenta, retorno ao cio, abortamento nas ovelhas e anemia hemolítica nos cordeiros com morte na primeira semana de vida. (CICERONI et al., 2000).

A sorovariedade hardjo é a mais freqüente em todo o mundo, portanto a maior causadora de problemas reprodutivos em ovelhas e de morte de cordeiros. Além dessa sorovariedade, também têm sido descritas outras sorovariedades, porém com menor freqüência, podendo ser destacada a presença das sorovares pomona, ballum, bratislava e grippotyphosa (ELLIS, 1983).

No Brasil, inquéritos sorológicos feitos por VIEGAS (1980); ELLIS (1994); VIEGAS (1994); CALDAS (1994); LANGONI et al. (1995); CALDAS et al.. (1995/96a) e CALDAS et al.. (1997/98) em ovinos e VASCONCELLOS et al. (1997) em bovinos, demonstraram a distribuição de Leptospira spp em vários estados brasileiros. No Estado do Rio Grande do Sul, onde a criação dessas duas espécies é comum, nos mesmos pastos e aguadas, a infecção por Leptospira spp provavelmente está estabelecida nos rebanhos.

O presente estudo teve como objetivo determinar a soroprevalência de aglutininas antiLeptospira spp em ovinos nas Mesorregiões Sudoeste e Sudeste Rio-Grandense no período de janeiro a março de 1999.

\section{MATERIAL E MÉTODOS}

As colheitas de amostras foram realizadas entre os meses de janeiro e março de 1999 em rebanhos de ovinos criados em 136 fazendas localizadas nas Mesorregiões Sudeste e Sudoeste Rio-Grandense na região Sul do Brasil. Para determinar a soroprevalência de aglutininas anti-Leptospira spp nos ovinos (Tabela 1), utilizou-se o modelo amostral preconizado, segundo o Centro Panamericano de Zoonoses (Cepanzo,1979). A prevalência esperada foi calculada em $12 \%$, erro amostral de $15 \%$ e com $95 \%$ de graus de confiança $(\mathrm{a}=1,96)$, resultando em uma amostra calculada de 1.252 animais, e foi aumentada em $30 \%$, totalizando 1.630 ovinos. Selecionaram-se aleatoriamente $50 \%$ dos municípios das duas Mesorregiões do estudo, resultando 10 municípios na Mesorregião Sudeste e 8 na Mesorregião Sudoeste (Tabela 1).

A população de ovinos dos município selecionados foi obtida dos arquivos do censo agropecuário do ano de 1996, (IBGE, 1998) e, para se obter o número de fazendas a serem trabalhadas nos municípios selecionados, foi realizada a divisão do total de ovinos criados nos 18 municípios (2.380.230), por 163 propriedades, resultando desta forma em intervalo amostral de 14.284 animais em cada município estudado. Em virtude da população ovina de cada fazenda sorteada ser muito diferenciada, a soroprevalência foi calculada ponderando-se os valores, segundo BENNETT et al. (1991).

Todos os ovinos sorteados ao acaso possuíam mais de um ano de idade, e no momento da colheita estavam clinicamente sadios e sem registros de histórico de doenças interferindo na reprodução. As amostras de sangue foram obtidas por venopunção jugular, e os soros congelados à temperatura de $-20^{\circ} \mathrm{C}$ e transportados em gelo, até o Laboratório de Zoonoses do Departamento de Medicina Veterinária Preventiva da UFMG, onde foram conservadas sob refrigeração até a realização dos testes.

Uma bateria antígenos vivos foram utilizadas formada por 18 amostras representando 17 sorovariedades de Leptospira spp, (Tabela 2), contendo aproximadamente $1-2 \times 10^{8}$ bactérias $\mathrm{mL}^{-1}$, livres de contaminantes e de auto-aglutinação. A escolha da bateria de antígenos foi feita a partir dos registros dos estudos sorológicos realizados em ovinos e acrescido das sorovariedades mais prevalentes encontradas em bovinos no Brasil.

Os exames sorológicos foram realizados pela técnica de MAT, segundo COLE et al. (1973), modificada no tempo de incubação para 60 minutos. As leituras das reações foram feitas diretamente na microplaca (NUNC F, Dinamarca) com microscópio, equipado com condensador seco de campo escuro, objetiva de longa distância Epiplan LD 10X/0.20, oculares E-pi 10X/20 e EP-L 10X/20, Axiolab®a

Os soros foram testados a partir da diluição de $1 / 100$, sendo considerados reagentes os soros que apresentaram 50\% ou mais de aglutinação. Após, foram diluídos na base 2 até determinação do título final.

\section{RESULTADOS}

Dos 18 municípios sorteados nas Mesorregiões sudeste e sudoeste Rio Grandense, em um apenas (Pelotas), não ocorreram amostras 
Tabela 1. Municípios selecionados para coletas de soros de ovinos nas Mesorregiões Sudeste e Sudoeste Rio-Grandense, RS. 1999.

\begin{tabular}{|c|c|c|c|c|c|c|c|}
\hline \multirow{2}{*}{\multicolumn{2}{|c|}{ Município }} & \multirow{3}{*}{$\begin{array}{c}\text { População de ovinos } \\
3.677\end{array}$} & \multicolumn{2}{|c|}{ Amostras previstas } & \multicolumn{2}{|c|}{ Amostras coletadas } & \multirow{3}{*}{$\begin{array}{c}\text { Mesorregião } \\
\text { Sudeste }\end{array}$} \\
\hline & & & \multirow{2}{*}{$\begin{array}{c}\text { Propriedades } \\
1\end{array}$} & \multirow{2}{*}{$\begin{array}{c}\text { Animais } \\
10\end{array}$} & \multirow{2}{*}{$\begin{array}{c}\text { Propriedades } \\
1\end{array}$} & \multirow{2}{*}{$\frac{\text { Animais }}{10}$} & \\
\hline 1 & Pelotas & & & & & & \\
\hline 2 & São Lourenço do Sul & 13.947 & 1 & 10 & 1 & 10 & Sudeste \\
\hline 3 & Hulha Negra & 16.563 & 1 & 10 & 1 & 10 & Sudoeste \\
\hline 4 & Pedro Osório & 25.204 & 2 & 20 & 2 & 20 & Sudeste \\
\hline 5 & Rio Grande & 30.348 & 2 & 20 & 2 & 20 & Sudeste \\
\hline 6 & São Francisco de Assis & 41.553 & 4 & 40 & 4 & 40 & Sudoeste \\
\hline 7 & Arroio Grande & 53.402 & 4 & 40 & 4 & 40 & Sudeste \\
\hline 8 & Canguçu & 53.628 & 4 & 40 & 4 & 40 & Sudeste \\
\hline 9 & Santana da Boa Vista & 62.259 & 4 & 40 & 4 & 40 & Sudeste \\
\hline 10 & São Borja & 78.149 & 5 & 50 & 5 & 50 & Sudoeste \\
\hline 11 & Lavras do Sul & 81.657 & 6 & 60 & 5 & 50 & Sudoeste \\
\hline 12 & Caçapava do Sul & 85.884 & 6 & 60 & 5 & 50 & Sudeste \\
\hline 13 & Piratini & 143.984 & 10 & 100 & 5 & 50 & Sudeste \\
\hline 14 & Bagé & 178.761 & 12 & 120 & 12 & 120 & Sudoeste \\
\hline 15 & Herval & 185.450 & 13 & 130 & 13 & 130 & Sudeste \\
\hline 16 & Uruguaiana & 386.717 & 25 & 250 & 24 & 240 & Sudoeste \\
\hline 17 & Alegrete & 423.446 & 30 & 300 & 30 & 300 & Sudoeste \\
\hline \multirow[t]{2}{*}{18} & Santana do Livramento & 463.491 & 32 & 320 & 14 & 140 & Sudoeste \\
\hline & Total & 2.328 .230 & 163 & 1630 & 136 & 1360 & \\
\hline
\end{tabular}

Fonte: Censo agropecuário 1996 (IBGE, 1998).

reagentes pela técnica da MAT e das 136 propriedades estudadas, em $113(83,09 \%)$ houve pelo menos uma amostra de soro reagente para uma das 17 sorovariedades de Leptospira spp utilizada na bateria de antígeno (Tabela 2).

Das 1.360 amostras de soro testadas, 466 $(34,26 \%)$ (IC 95\% 29,74 a 38,78\%) apresentaram reação de aglutinação positiva, com títulos de anticorpos superiores a 100, para várias sorovariedades. Foi considerada aquela que apresentava maior título sorológico (Tabela 3).

Neste trabalho, foram utilizadas duas amostras da sorovariedades hardjo, sorogrupo serjoe (hardjoprajitno, padrão e hardjo Amostra Norma), isolada na Escola de Veterinária da UFMG. Esses sorovares foram os mais prevalentes representando, $30,10 \%$ do total de reações (IC 95\%, 16,96\% a 44,23\%). Em ordem decrescente, a segunda mais prevalente foi a sorovariedade sentot com 152 reações $(16,8 \%$ IC, $95 \%, 7,15$ a $26,45 \%$ ) seguida pela sorovar fortbragg com 73 reações $(6,3 \%$ IC $95 \% 3,72$ a $8,88 \%)$.

\section{DISCUSSÃO}

Os resultados obtidos neste trabalho, embora semelhante àqueles encontrados por LANGONI et al., (1995) no Estado de São Paulo, e menores do que aqueles relatados por VIEGAS (1980), VIEGAS (1994), CALDAS (1994), CALDAS et al. (1995/ 96a) e CALDAS et al. (1997/98), na Bahia, mesmo assim, não podem ser comparados pois enquanto a maioria dos autores trabalhou com amostras de animais com problemas reprodutivos, sem representação estatística, neste, foram aplicados métodos de amostragem probabilística, além do local e o período de pesquisa serem diferentes. Ainda há que se ponderar que a bateria dos antígenos utilizados, tanto neste trabalho como nos citados acima não era a mesma.

A prevalência ponderada de 48,7\% (IC 95\%, 35,4-62,0\%) encontrada nas Mesorregiões Sudeste e Sudoeste Rio-Grandense e em 17 dos 18 municípios probabilisticamente sorteados, evidenciam a larga dispersão da Leptospiras spp na região e corroboram com o trabalho de SANTA ROSA \& PESTANA DE CASTRO (1963), que já naquela época alertaram os criadores e técnicos gaúchos sobre a possibilidade da leptospirose ser um dos grandes responsáveis por enormes prejuízos econômicos da ovinocultura do Sul, principalmente ligado aos episódios reprodutivos e mortalidade de recém-nascidos, o que não foi objetivo deste trabalho. Por outro lado, a não detecção de anticorpos em animais do município de Pelotas não indica que esse seja livre da doença, pois as condições 
Tabela 2 - Sorovariedades de Leptospira spp, utilizados como antígeno para técnica de aglutinação microscópica.

\begin{tabular}{|c|c|c|c|}
\hline Sorogrupos & Sorovariedades & Espécies & Amostras \\
\hline \multirow{2}{*}{ AUSTRALIS } & australis & L. interrogans & BALLICO \\
\hline & bratislava & L. interrogans & JEZ BRATISLAVA \\
\hline \multirow{2}{*}{ AUTUMNALIS } & autumnalis & L. interrogans & AKIYAMY H \\
\hline & fortbragg & L. noguchii & FORT BRAGG \\
\hline BALLUM & castellonis & L. borgpetersenni & CASTELLON 3 \\
\hline CANICOLA & canicola & L. interrogans & HOND UTRECHT IV \\
\hline DJASIMAN & sentot & L. interrogans & SENTOT \\
\hline GRIPPOTYPHOSA & grippotyphosa & L. interrogans & MOSKVA V \\
\hline HEBDOMADIS & hebdomadis & L. interrogans & HEBDOMADIS \\
\hline ICTEROHAEMORRAGIAE & icterohaemorrhagiae & L. interrogans & RGA \\
\hline MINI & $\operatorname{mini}$ & L. santarosai & SZWAJIZAK \\
\hline POMONA & pomona & L. interrogans & POMONA \\
\hline PYROGENES & pyrogenes & L. interrogans & SALINEM \\
\hline \multirow{4}{*}{ SEJROE } & sejroe & L. borgpetersenni & M 84 \\
\hline & hardjo & L. interrogans & HARDJOPRAJITNO \\
\hline & & & HARDJO (Norma) \\
\hline & wolffi & L. interrogans & 3705 \\
\hline TARRASOVI & tarrasovi & L. borgpetersenni & PEREPELECIN \\
\hline
\end{tabular}

locais, de baixadas alagadas são propícias à manutenção da doença. No entanto, a colheita de somente, 10 animais de um único rebanho, devido ao delineamento experimental utilizado, pode ter sido um equívoco metodológico.

Verificou-se uma variação nos títulos aglutinantes de 100 até 3.200 , das 774 amostras reagentes. Desses, $634(81,91 \%)$ eram menores ou iguais a 200 e, $140(18,19 \%)$ iguais ou superiores a 400 . Isso mostra uma situação epidemiológica grave, pois demonstra que a infecção está ativa o que confirma os achados de ELLIS (1994) que encontrou animais recentemente infectados com título variando entre 100 e 200. Neste trabalho, o maior título encontrado foi de 3.200, sugerindo infecção grave.

A sorovariedade hardjo é largamente distribuída entre animais domésticos no Brasil e com certa freqüência são encontrados em soros de animais com título de anticorpos superiores a 100, sem no entanto, estes apresentarem sinais clínicos clássicos de leptospirose. Entretanto, essa sorovariedade pode determinar que o animal apresenta sinais clínicos mais graves, como transtornos reprodutivos, natimortos e crias fracas com altas taxas de mortalidade nos primeiros dias de nascidos, o que já foi comprovado por ELLIS (1983). Isso pode levar muitas vezes a diagnósticos incorretos e consequentemente com medidas de controle inadequadas. Embora o trabalho tenha seguido o procedimento tradicional de partir de uma diluição de 1/100 e de considerar os animais reagentes com título de 100 ou maior, BLACKMORE et al. (1982) afirmam que animais com título $\leq 50$ podem ser considerados infectados e apresentar transtornos reprodutivos. Desta forma, estes resultados podem estar subestimados.

É conhecido por estudos em bovinos, que a sorovariedade mais prevalente no Brasil é a hardjo (VASCONCELLOS et al., 1997). Nesta pesquisa em ovinos, também a mesma sorovariedade foi a mais prevalente e coincidentemente com as maiores freqüências na Mesorregião Sudoeste, onde estão localizados as grandes propriedades e as maiores concentrações de ovinos e bovinos.

Embora a sorovariedade hardjo seja a mais prevalente nesse trabalho, foram utilizadas na bateria, duas variantes, uma considerada padrão, hardjoprajitno e uma amostra hardjo (Norma) isolada por MOREIRA (1994) em bovinos do Estado de Minas Gerais. Os resultados obtidos evidenciaram a necessidade de estudos sobre as baterias de antígenos utilizados em diagnóstico de rotina. Dos 233 animais reagentes para a sorovariedade hardjo, $110(8,09 \%)$ reagiram só para a variante (Norma), 23 $(1,69 \%)$ só para a hardjoprajitno e $100(7,35 \%)$ para as duas variantes concomitantemente. Esse fato sinaliza a necessidade de pesquisa e isolamento de novos sorovares ocorrentes nos rebanhos, pois os antígenos mantidos indefinidamente em meios de cultura com repiques freqüentes, pode selecionar variantes que diferem daquelas encontradas na natureza, como parece ser o que ocorreu neste trabalho.

Ciência Rural, v.34, n.2, mar-abr, 2004. 
Tabela 3 - Distribuição da soroprevalência de aglutininas antiLeptospira spp em 1.360 soros ovinos das Mesorregiões Sudeste e Sudoeste Rio-Grandense, 1999.

\begin{tabular}{lccc}
\hline Sorovariedades & Ovinos & Prevalência $^{\mathrm{a}}$ & $95(\%) \mathrm{IC}^{\mathrm{b}}$ \\
\hline hardjo & 233 & 30,66 & $16,96-44,23$ \\
Amostra (Norma) & 210 & 28,40 & $14,54-42-26$ \\
hardjoprajitno & 23 & 1,69 & $0,00-3,45$ \\
sentot & 152 & 16,8 & $7,15-26,44$ \\
fortbragg & 73 & 6,3 & $3,71-8,88$ \\
wolffi & 39 & 4,7 & $2,21-7,18$ \\
pyrogenes & 25 & 1,8 & $0,00-3,74$ \\
australis & 21 & 1,5 & $0,40-2,60$ \\
pomona & 20 & 1,6 & $0,00-3,55$ \\
sejroe & 19 & 2,2 & $0,00-4,64$ \\
castellonis & 18 & 1,8 & $0,32-3,27$ \\
hebdomadis & 17 & 1,25 & $0,00-2,64$ \\
Icterohaemorrhagiae & 16 & 1,10 & $0,00-1,03$ \\
grippotyphosa & 9 & 0,60 & $0,00-1,45$ \\
canicola & 8 & 0,6 & $0,00-1,28$ \\
tarassovi & 7 & 0,6 & $0,00-1,32$ \\
bratislava & 4 & 0,2 & $0,00-0,48$ \\
autumnalis & 3 & 0,1 & $0,00-0,30$ \\
mini szwajizak & 0 & 0,0 & $0,0-0,0$ \\
\hline
\end{tabular}

a- Prevalência ponderada calculada segundo BENNNET et al.,(1991),(48,7\% IC 95\% 35,4\%-62,00\%).

b- Intervalo de Confiança $95 \%$.

Outro fato importante é a controvérsia entre pesquisadores sobre achados de pesquisa. MOREIRA (1994) afirma que é raro ocorrer duas ou mais sorovariedades infectando o mesmo rebanho, enquanto ROTH \& GALTON (1960) sugerem a possibilidade da hardjo e sejroe ocorrerem simultaneamente, o que parece ter sido esse caso.

Sendo a sorovariedade hardjo a mais prevalente nos rebanhos bovinos brasileiros, é a primeira vez que sua presença é relatada em ovinos no Estado do Rio Grande do Sul o que era esperado pelo tipo misto de criação ovino/bovinos em regime extensivo.

De fundamental importância, constatou-se o fato de que a cepa Norma, isolada no estado de Minas Gerais por MOREIRA (1994) tem maior prevalência no Estado do Rio Grande do Sul, superando a amostra padrão hardjoprajitno. Isso indica que as vacinas usuais do mercado, feitas com amostras padrões, podem não estar protegendo dentro da margem de segurança ou ainda estar permitindo resultados não confiáveis, quando se usa só amostra padrão na Bateria de antígenos.

Neste trabalho, foi a primeira vez no Brasil que foi relatada a presença da sorovariedade sentot em animais domésticos, com o agravante de ser o segundo sorovar em prevalência com (16,8\%) (IC 95\% 7,15 a 26,45\%) nos animais estudados. O único relato desse sorovar no Brasil foi em um paciente humano no Estado de São Paulo (ALTÉRIO \& CUNHA, 1966). Isso provavelmente é porque essa sorovariedade não está incluída nas baterias de antígenos usualmente utilizados e não pela sua ausência no ambiente. Esse achado corrobora com os comentários de FAINE (1982) indicando a necessidade de isolamento e tipificação de novos sorovares, sempre que são detectados a primeira vez em uma região.

O ambiente e o manejo produtivo de bovinos e ovino no Estado do Rio Grande do Sul favorecem a manutenção e disseminação da doença. Embora os bovinos sejam os hospedeiros principais do sorovar hardjo, o estreito convívio dos ovinos com bovinos, principalmente nos grandes rebanhos, propicia essa transmissão entre espécies e, a partir de ovinos infectados, pode ocorrer disseminação entre os da sua espécie. Outro modo freqüente de transmissão poderá ser a alimentação de cordeiros com leite de vaca, prática usual que ocorre em casos especiais de parição múltipla, morte da mãe ou animais fracos que não acompanham o rebanho. Esses e outros fatores são amplamente discutidos por outros autores como SIMPSON \& DONE (1989), ELLIS (1994), DRAGHIDEBERNITEZ\& BIOTTI DECARCERES (1998).

No Estado do Rio Grande do Sul, que tem o maior rebanho ovino do Brasil, com grande tradição pecuária, até o presente momento não havia notificação por estudo sorológico das leptospiroses como uma causa possível causa de transtorno reprodutivos e mortalidade de animais jovens. Um dos poucos trabalhos para identificar a causa morte de cordeiros, foi feito, por histopatologia, por OLIVEIRA \& BARROS (1982), os quais, não consideraram as leptospiroses como causa das mortesou o possível envolvimento de agentes infecciosos. Os resultados deste trabalho sugerem a necessidade de ser estabelecido e implantado um programa sanitário na pecuária de ovinos visando determinar os agentes infeciosos mais prevalentes e propor medidas de controle.

\section{CONCLUSÃO}

O rebanho ovino das Mesorregiões Sudeste e Sudoeste Rio-Grandense, está exposto a várias sorovariedades de Leptospira spp, sendo a mais prevalente a sorovariedade hardjo. Há necessidade da elaboração de programas de controle da doença por meio da produção e o uso de vacinas homológas específicas para os ovinos da região, e a adoção de 
uma série de medidas profiláticas entre elas incluem a vacinação sistemática de todos os ovinos do plantel. Realizar novas pesquisas da Leptospira spp no Estado do Rio Grande do Sul, estendendo os estudos também no homem e outras espécies de animais, destinando esforços para o isolamento a identificação de amostras.

\section{FONTES DE AQUISIÇÃO}

${ }^{a}$ Schott-Zeiss do Brasil Ltda Av. Nações Unidas 21711, 04795-100, São Paulo, SP.

\section{AGRADECIMENTOS}

À FEP/MVZ Coordenação Preventiva pelo apoio financeiro, à CAPES/PICDT (GPH), ao CNPq pelas bolsas concedidas, à BRAZEISS Representações LTDA pelo empréstimo do microscópio, à Secretaria da Agricultura e Abastecimento do Estado do Rio Grande do Sul e ao Antônio Benjamim de Paula pelo auxilio técnico laboratorial.

\section{REFERÊNCIAS BIBLIOGRÁFICAS}

ALTÉRIO, D.L; CUNHA, J.C. Human leptospirosis caused by Leptospira sentot. Revista Clinica da Faculdade de Medicina São Paulo, v.21. n.4, p.199-201, 1966.

BENNETT, S. et al. A simplified method for cluster-sample surveys of health in developing countries. Rapp Trimestral Statistical Sanitari Mond, v.44, n.3, p.98-106, 1991.

BLACKMORE, D.K. et al. The epidemiological interpretation of serological response to leptospiral serovars in sheep. New Zealand Veterinary Journal, v.30, n.4, p.38-42, 1982.

CALDAS, E.M. et al. Nota sobre atualização de diferentes antígenos de Leptospira para a detecção de anticorpos nos de animais domésticos. Arquivos da Escola de Veterinária da Universidade Federal da Bahia, v.17, n.1, p.1-7, 1994.

CALDAS, E.M. et al. Estudo comparativo entre estirpes de L. interrogans e L. biflexa no diagnóstico de triagem de leptospirose em animais. Arquivos da Escola de Veterinária da Universidade Federal da Bahia, v.18, n.1, p.127-147, 1995/96a.

CALDAS, E.M. et al. Estudo comparativo entre o teste da macroaglutinação e a soroaglutinação microscópica, utilizando antígenos de $\boldsymbol{L}$. interrogans e $\boldsymbol{L}$. biflexa no diagnóstico rápido da leptospirose em animais. Arquivos da Escola de Veterinária da Universidade Federal da Bahia, v.19, n.1, p.155-177, 1997/98.

CENTRO PANAMERICANO DE ZOONOSES, Ramos Mejia. Procedimientos para estudos de prevalência por muestreo. Buenos Aires : CEPANZO, 1979. 39p (Nota Técnica 18).

CICERONI, L. et al. Prevalence of antibodies to Leptospira serovars in sheep and goats in Alto Adige-South Tyrol. Journal Veterinary Medicine, v.47, n.5, p.217-223, 2000.
COLE, J.R. et al. Improved microtecnique for the leptospiral microscopic aglutination test. Applied Microbiology, v.25, n.6, p.976-980, 1973.

DRAGHI DE BENÍTEZ, M.; BIOTTI DE CARCERES, G.M. Aislamiento de Leptospira interrogans en un cordero da la provincia de Corrientes (Republica da Argentina). Revista Medicina Veterinária, v.79, n.3, p.224-225, 1998.

ELLIS, W.A. et al. Possible involviment of leptospires in abortion, stilbirths and neonatal deaths in sheep. Veterinary Record, v.26, n.12, p.291-293, 1983.

ELLIS, W.A. Leptospirosis as a cause of reproductive failure. Veterinary Clinics of North America: Food Animal Practice, v.10, n.3 p.463-478, 1994.

FAINE, S. Guidelines for the control of leptospirosis. Genebra : World Health Organization, 1982. 171p. (Ofset publication, 67).

FAINE, S. et al. Leptospira and leptospirosis. 2.ed. Austrália : MediSci, 1999. 272p.

INSTITUTO BRASILEIRO DE GEOGRAFIA E ESTATISTICA. Censo Agropecuário, 1996. Disponível em: <www.sidra.ibge.gov.br/cgr-bm/prtabr, Acesso 25/10/1998>.

LANGONI, H. et al. Pesquisa de aglutininas anti-leptospiras em soros ovinos do Estado de São Paulo, Brasil, utilizando provas de macroaglutinação em placa e soroaglutinação microscópica. Revista Brasileira de Medicina Veterinária, v.17, n.6, p.264-268, 1995.

MOREIRA, E.C. Avaliação de métodos para erradicação de leptospiroses em bovinos leiteiros. 1994. 94f. Tese (Doutorado em Ciência Animal) - Escola de Veterinária da UFMG.

OLIVEIRA, A.C.; BARROS, S.S. Mortalidade perinatal em ovinos no município de Uruguaiana, Rio Grande do Sul. Pesquisas Veterinária Brasileira, v.2, n.1, p.1-7, 1982.

ROTH, E.E.; GALTON, M.M. Isolation and identification of Leptospira hardjo from cattle in Lousiana. Americam Journal Veterinary Research, v.21, n.5, p. 411-427, 1960.

SANTA ROSA, C.A.; PESTANA de CASTRO, A.F. Presença de aglutininas antileptospiras em soro de ovinos e caprinos no Estado de São Paulo. Arquivos Instituto de Biologia, v.30, p.93-98, 1963.

SIMPSON, V.R.; DONE, S. Leptospira hardjo and lambs received cows colostrum. Veterinary Record, v.124, n.17, p.469, 1989.

VASCONCELLOS S.A. Leptospirose bovina. Níveis de ocorrência e sorotipos predominantes em rebanhos dos Estados de Minas Gerais, São Paulo, Rio de janeiro, Paraná e Rio Grande do Sul. Período de Janeiro a Abril de 1996. Arquivos do Instituto biológico de São Paulo, v.64, n.2, p.7-15, 1997.

VIEGAS, E.A. et al. Aglutininas anti-Leptospira em hemosoro de caprinos e ovinos, no Estado da Bahia. Arquivos da Escola de Veterinária da Universidade Federal da Bahia, v.5, n.1, p.20-34, 1980.

VIEGAS, E.A. et al. Emprego de estirpes de Leptospira biflexa na prova de soroaglutinação microscópica aplicada ao diagnóstico da leptpsirose caprina e ovina. Brazilian Journal Veterinary Animal Science v.31. n.1. p.25-30, 1994. 